\title{
MicroRNA-92a overexpression promotes the osteogenic differentiation of bone mesenchymal stem cells by impeding Smad6-mediated runt-related transcription factor 2 degradation
}

\author{
$\mathrm{XU}_{\mathrm{YAN}^{1}}{ }^{*}, \mathrm{HAO}_{\mathrm{WANG}}{ }^{2 *}, \mathrm{YUFEI} \mathrm{LI}^{3}, \mathrm{YUXIN} \mathrm{JIANG}^{4}$, QINGDONG SHAO ${ }^{1}$ and WEIDONG XU ${ }^{1}$ \\ ${ }^{1}$ Department of Orthopedics, 455th Hospital of PLA, Shanghai 200052; ${ }^{2}$ Teaching Center of Experiment Medicine, \\ Shanghai Medical College, Fudan University, Shanghai 200032; ${ }^{3}$ Department of Plastic Surgery, 455th Hospital of PLA, \\ Shanghai 200052; ${ }^{4}$ School of Medicine, Dalian Medical University, Dalian, Liaoning 116044, P.R. China
}

Received November 2, 2017; Accepted February 14, 2018

DOI: $10.3892 / \mathrm{mmr} .2018 .8829$

\begin{abstract}
Bone mesenchymal stem cells (BMSCs) are an important source of stem cells for tissue repair and regeneration; therefore, understanding the mechanisms that regulate stem cell differentiation in a specific lineage is critical. Runt-related transcription factor 2 (Runx2) is a bone-specific transcription factor that serves an important role in promoting osteogenic differentiation. However, Runx2 protein levels are regulated by the ubiquitin-proteasome pathway. Previous research has identified that Smad6 can interact with Runx2 and enhance Smurf1-induced Runx2 degradation in a ubiquitin-proteasome-dependent manner. Bioinformatics analysis demonstrated that miR-92a can target Smad6. To characterize the regulatory effect of miR-92a on osteogenic differentiation of BMSCs and assess the interactive association between Smad6 and miR-92a, BMSCs were obtained from mice and miR-92a or Smad6 overexpression vectors were constructed. Reverse transcription-quantitative polymerase chain reaction (RT-qPCR) and western blots were used to analyze the expression of miR-92a and Smad6, and the luciferase reporter assay was used to examine the interaction between miR-92a and Smad6. BMSCs were induced in osteogenic differentiation media for 21 days. The alkaline phosphatase activity was assessed and Alizarin Red histochemical staining was also performed. The results suggested that the expression of miR-92a suppressed Smad6-mediated Runx2 degradation by direct integration with the 3'-UTR of Smad6 mRNA, which was confirmed by a luciferase reporter
\end{abstract}

Correspondence to: Dr Qingdong Shao or Dr Weidong Xu, Department of Orthopedics, 455th Hospital of PLA, 338 West Huaihai Road, Shanghai 200052, P.R. China

E-mail: shaoqingdong@foxmail.com

E-mail: xuweidongch@126.com

*Contributed equally

Key words: microRNA-92a, Smad6, bone mesenchymal stem cells, osteogenic differentiation, runt-related transcription factor 2 assay. In addition, the expression of miR-92a promoted the osteogenic differentiation of BMSCs. However, the regulatory effect of miR-92a was inhibited by overexpression of Smad6. Taken together, the results suggest that miR-92a expression inhibits the osteogenic differentiation of BMSCs by targeting Smad6.

\section{Introduction}

Stem cells, which are characterized by their self-renewal and multilineage differentiation capacity, have recently attracted attention for their potential medicinal applications (1). The process of osteogenic differentiation is known to involve many regulatory transcriptional factors. Runt-related transcription factor 2 (Runx2) has been identified as a key transcription factor in the early stage of osteogenesis $(2,3)$. Runx 2 may regulate osteogenic differentiation by targeting several bone-specific transcriptional factors $(4,5)$. Runx 2 is also an important regulator of chondrocyte differentiation and maturation. Evidence showing an alteration of chondrocyte maturation in long bones of Runx2 null mutant mice, as well as in cell culture studies, indicate that Runx 2 is a positive regulator of chondrocyte differentiation (6-8). Under-expression of Runx2 in proliferating chondrocytes induces chondrocyte hypertrophy and partially rescues the chondrocyte phenotype of Runx2 null mutant mice $(9,10)$. Selective inactivation of Runx 2 in chondrocytes results in severe shortening of the limbs due to a disturbance in chondrocyte differentiation (11). Fan et al have shown that the CREB-Smad6-Runx2 axis is involved in osteogenesis dysfunction of bone mesenchymal stem cells (BMSCs). Smad6 can interact with Runx2 and enhance Smurf1-induced Runx2 degradation in a ubiquitin-proteasome-dependent manner $(12,13)$, suggesting that suppressed expression of Smad6 may promote osteogenic differentiation in BMSCs.

How to effectively promote BMSC osteogenic differentiation has become a core issue in bone repair or regeneration. Bioinformatics analysis has found that miR-92a can target Smad6 by direct integration with the 3'-UTR of Smad6 mRNA (http://www.targetscan.org/), suggesting that miR-92a expression of may promote the osteogenic differentiation of BMSCs by targeting Smad6. 
To define the function and regulatory mechanisms of miR-92a in the osteogenic differentiation of BMSCs, BMSCs were obtained from mice and miR-92a or Smad6 overexpression vectors were constructed. Reverse transcription-quantitative polymerase chain reaction (RT-qPCR) and western blots were used to analyze miR-92a and Smad6 expression, and a luciferase reporter assay was used to examine the interaction between miR-92a and Smad6. BMSCs were induced in osteogenic differentiation media for 21 days and then analyzed by alkaline phosphatase (ALP) activity and Alizarin Red histochemical staining. The results may improve our knowledge of the epigenetic mechanisms governing BMSC osteoblastic lineage differentiation, which would benefit the development of bone tissue engineering or cell therapy based on BMSCs.

\section{Materials and methods}

Ethics statement. BALB/C mice (6-8 weeks old) were obtained from the Shanghai SLAC Laboratory Animal Co., Ltd. (Shanghai, China). All animal studies were performed in accordance with the Guide for the Care and Use of Laboratory Animals. All study protocols were approved by the Scientific Research Projects Approval Determination of the Ethics Committee of the 455th Hospital of PLA.

Isolation and characterization of BMSCs. BMSCs were harvested from 6- to 8-week-old mice as described previously (14). Mice were euthanized and both femurs and tibiae were aseptically removed. Then the ends of the femurs and tibiae were cut and the bone marrow was flushed out and diluted 1:2 with phosphate buffer solution and loaded in a $5 \mathrm{ml}$ Percoll (density, 1.077; Pharmacia Biotech, Uppsala, Sweden). Cells were harvested from the interface after centrifugation at 2,000 x g for $20 \mathrm{~min}$ and washed with Dulbecco's modified Eagle's medium (DMEM; Gibco; Thermo Fisher Scientific, Inc., Waltham, MA, USA). They were then resuspended in low-glucose DMEM containing $10 \%$ fetal bovine serum (HyClone; GE Healthcare Life Sciences, Logan, UT, USA), $100 \mathrm{U} / \mathrm{ml}$ penicillin, $100 \mathrm{~g} / \mathrm{ml}$ streptomycin, and incubated at $37^{\circ} \mathrm{C}$. Cells of the 2 nd to 5 th passages were used in this study.

For BMSC characterization, cells were seeded again at 1:4 density ratios and tested by immunofluorescence, with positive results for CD29, CD44, CD90, and CD105, but negative results for hematopoietic markers such as CD34 and CD45.

For multiple differentiation capacity assays, BMSCs were cultured for 3 weeks in either adipogenic differentiation media or osteogenic differentiation media (Cellular Engineering Technologies Inc., Coralville, IA, USA). Oil-red O stain or ALP were used to identify the multiple differentiation capacities of BMSCs.

Transfection of cells with the miR-92a mimics vector, Smad6 overexpression vector or treatment with miR-92a inhibitor. For miR-92a overexpression, the miR-92a mimic or corresponding negative control (miR-NC) were purchased from GenePharma (Shanghai, China). BMSCs were transfected with either the miR-92a mimic or miR-NC at a final concentration of $50 \mathrm{nM}$ using Lipofectamine ${ }^{\circledR} 2000$ (Invitrogen; Thermo Fisher Scientific, Inc.) according to the manufacturer's protocol. Cells were used for miR-92a expression analysis or other experiments after $48 \mathrm{~h}$ of transfection. For miR-92a inhibition, BMSCs were treated with a miR-92a inhibitor (Invitrogen; Thermo Fisher Scientific, Inc.) for $48 \mathrm{~h}$, then BMSCs were collected for further experiments.

For Smad6 overexpression, human Smad6 cDNA with the 3'-UTR was cloned into a pMSCV-hygro vector. The primers corresponded to the NCBI reference sequence (AF202257.3) and were as follows: Forward, 5'-CAGAGCTCATGTTCA GGTCTAAACG-3' and reverse, 5'-GGTCTAGACTATCTG TGGTTGTTGAGT-3'. The Smad6 cDNA was inserted into a pMD18-T Simple Vector (Takara Bio, Inc., Otsu, Japan) to form pMD18-T-Smad6. After sequencing, the recombinant segment of the correct clone was digested by BamHI and $\mathrm{XbaI}$ (Takara Bio, Inc.). The recombinant segment was inserted into pMSCV-hygro after digestion by the same two restriction endonucleases. The pMSCV-hygro-Smad6 clones were sequenced and the correct clones were amplified and identified by restriction enzyme digestion.

$R T$-qPCR for the detection of miR-92a. Total RNA was isolated using TRIzol ${ }^{\circledR}$ reagent (Invitrogen; Thermo Fisher Scientific, Inc.). RT was performed using the RT-PCR system (Promega, Shanghai, China). RT-qPCR was performed in a final reaction volume of $20 \mu \mathrm{l}$ using $\mathrm{SYBR}^{\circledR}$-Green I Supermix (Takara Biotechnology Co., Ltd., Dalian, China) according to the manufacturer's protocol. All reactions were run in triplicate on a iCycler IQ Multicolor Detection System (Bio-Rad Laboratories, Inc., Hercules, CA, USA) with the following cycling parameters: $95^{\circ} \mathrm{C}$ for $10 \mathrm{sec}$ followed by 40 cycles of $94^{\circ} \mathrm{C}$ for $15 \mathrm{sec}$, annealing at $55^{\circ} \mathrm{C}$ for $30 \mathrm{sec}$, and a final extension at $70^{\circ} \mathrm{C}$ for $30 \mathrm{sec}$. All quantifications were normalized to the level of human U6 snRNA in the same reaction. The comparative quantification $(\mathrm{Cq})$ cycle method $\left(2^{-\Delta \Delta \mathrm{Cq}}\right)$, which compares differences in $\mathrm{Cq}$ values between common reference RNA and target gene RNA, was used to obtain the relative fold-changes in gene expression. The miR-92a primers for PCR were designed by GenePharma.

MTT assay. Cell toxicity was measured with an MTT assay to detect NADH dependent dehydrogenase activity. Fifty microliters of MTT solution $(5 \mathrm{mg} / \mathrm{ml})$ in $1 \mathrm{X}$ phosphate-buffered saline were directly added to the cells, which were then incubated for $2 \mathrm{~h}$ to allow MTT to metabolize to formazan. Absorbance was measured at a wavelength of $540 \mathrm{~nm}$ using an ELISA reader (Beckman Coulter, Inc., Brea, CA, USA).

Osteoblastic differentiation. To identify the effect of miR-92a and Smad6 on osteoblastic differentiation, BMSCs were transfected with miR-92a or the Smad6 overexpression vector or treated with a miR-92a inhibitor after BMSCs reached $70-80 \%$ confluence in basal medium. Then the medium was changed to osteogenic differentiation media to allow the cells to differentiate into osteoblasts and then cultured for 21 days, with fresh culture medium changed every 3 days.

ALP activity assay and Alizarin red staining. BMSCs were seeded into 96 -well plates at a density of $2 \times 10^{3}$ cells/well and cultured in routine medium or mineralization-inducing medium (MM) containing $\alpha$-MEM, $10 \%$ fetal bovine serum, $100 \mathrm{U} / \mathrm{ml}$ penicillin, $100 \mu \mathrm{g} / \mathrm{ml}$ streptomycin, $100 \mu \mathrm{M}$ ascorbic 
acid, $2 \mathrm{mM}$ 2-glycerophosphate, and $10 \mathrm{nM}$ dexamethasone. The ALP activity assay was performed using an ALP activity kit (Sigma-Aldrich; Merck KGaA, Darmstadt, Germany) and the activity was normalized to total protein content in the cell. Alizarin red staining was performed after treatment on day 21. Calcified nodules were stained using $10 \%$ cetylpyridinium chloride in $10 \mathrm{mM}$ sodium phosphate ( $\mathrm{pH}$ 7.0). Calcium concentration was determined by measuring the absorbance at $526 \mathrm{~nm}$ with a universal microplate reader (BioTek Instruments, Inc., Winooski, VT, USA). This experiment was performed in triplicate and the results are presented as the mean \pm standard deviation (SD).

Luciferase reporter assay. To construct luciferase reporter vectors, the 3'-UTR of Smad6 cDNA fragments containing the predicted miR-92a binding sites were amplified by PCR and subcloned downstream of the luciferase gene in the PYr-MirTarget luciferase vector (Ambion; Thermo Fisher Scientific, Inc.). The 3'-UTR of Smad6 (containing the binding sites for miR-92a) was amplified from a cDNA library with the following primers: Forward, 5'-CTCGAGCGTAATTAT TTATATATGGTGCAATGTG-3' and reverse, 5'-GCGGCC GCGGTCTGGGCATTAATATTT-3'. The mutant 3'-UTR of Smad6 (in which six nucleotides were mutated in the binding sites) was amplified using the following primer sequences: Forward, 5'-CTCGAGCGTAATTATTTATATATGCAC GTTGTG-3' and reverse, 5'-GCGGCCGCGGTCTGGGCA TTAATATTT-3'.

For luciferase assays, HEK293T cells were cultured in 24-well plates and co-transfected with $50 \mathrm{ng}$ of the corresponding vectors containing firefly luciferase together with $50 \mathrm{ng}$ of miR-92a or the control. Transfection was performed using Lipofectamine ${ }^{\circledR} 2000$ reagent (Invitrogen; Thermo Fisher Scientific, Inc.). At $48 \mathrm{~h}$ post-transfection, the relative luciferase activity was calculated by normalizing the firefly luminescence to the Renilla luminescence using the Dual-Luciferase Reporter Assay (Promega Corporation, Madison, WI, USA) according to the manufacturer's instructions.

Western blot analysis. Western blot analysis was carried out using cell lysates in urea buffer ( $8 \mathrm{M}$ urea, $1 \mathrm{M}$ thiourea, $0.5 \%$ CHAPS, $50 \mathrm{mM}$ dithiothreitol, $24 \mathrm{mM}$ spermine). Protein fractions were prepared using cytoplasmic extraction reagents (Pierce; Thermo Fisher Scientific, Inc.) following manufacturer's protocols. GAPDH was used as a loading control. Samples (40 $\mu \mathrm{g}$ total protein) were separated on SDS-PAGE and transferred to nitrocellulose membranes (EMD Millipore, Billerica, MA, USA). After blocking in 5\% nonfat milk for $1 \mathrm{~h}$, the membranes were incubated with primary antibodies against Smad6 (1:1,000), Runx2 (1:200), osteopontin (OPN, 1:200), osteocalcin (OCN, 1:200), osterix (OSX, 1:200) and GAPDH $(1: 2,000)$, respectively, at $4^{\circ} \mathrm{C}$ overnight. After washing, the membranes were incubated with horseradish peroxidase-conjugated secondary antibodies for $1 \mathrm{~h}$ at room temperature. Signals were detected using an ECL detection system (GE Healthcare, Aurora, OH, USA) and analyzed with ImageJ 1.42q software (National Institutes of Health, Bethesda, MD, USA).

Statistical analysis. Results are expressed as the mean \pm SD. Statistical significance was evaluated by one-way analysis of variance followed by the Tukey-Kramer multiple comparison
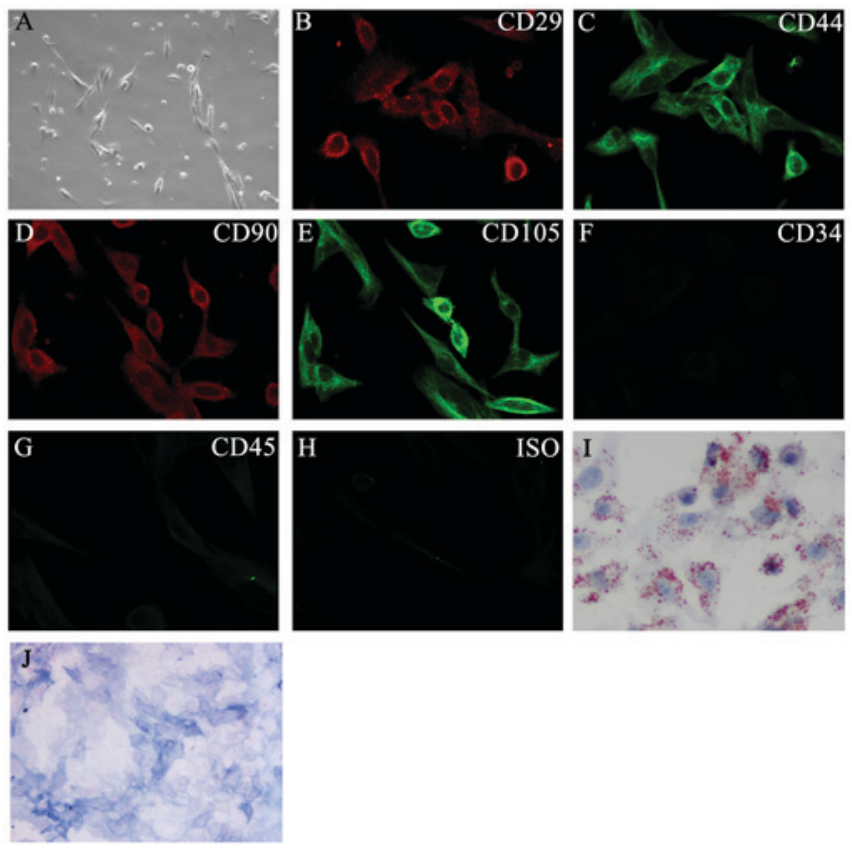

Figure 1.(A) Bone mesenchymal stem cells (BMSCs) showing a typical cobblestone-like morphology. Magnification, X200x. (B-H) Immunofluorescence analysis of stem cells markers in isolated BMSCs. Detection of cell surface markers by immunofluorescence staining with antibodies against (B) CD29, (C) CD44, (D) CD90, (E) CD105, (F) CD34, and (G) CD45 labeled with FITC (green color) or PE (red color). (H) A negative FITC IgG isotype control was included. Magnification (200x). (I) Oil-red O staining. Magnification, x200. (J) ALP staining. Magnification, x200.

test and by Student's t-test. $\mathrm{P}<0.05$ was considered to indicate a statistically significant difference.

\section{Results}

Isolation and identification of BMSCs with stem cell markers. In order to identify if the expression of miR-92a can promote osteogenic differentiation in BMSCs, BMSCs were isolated from mice. The predominant cells after 9-10 days of culture were morphologically homogeneous and spindle-shaped, and most of them were mesenchymal stem cells (Fig. 1A). Isolated BMSCs were labeled with different cell surface markers to verify that the isolated cells were truly mesenchymal stem cells. The results show that BMSCs were positive for the MSC markers CD29, CD90, CD44, and CD105 and negative for the endothelial markers CD34 and CD45 (Fig. 1B-H), indicating that the isolated cells were BMSCs. Oil-red O staining and ALP activity showed the adipogenic (Fig. 1I) and osteogenic differentiation (Fig. 1J) potential of BMSCs under adipogenic or osteogenic induction conditions.

miR-92a expression promotes osteogenic differentiation of $B M S C s$. To identify if miR-92a expression can promote the osteogenic differentiation of BMSCs, miR-92a overexpression and miR-92a mimic vectors were constructed and transfected into BMSCs and cultured for $48 \mathrm{~h}$. For the inhibition of miR-92a expression, BMSCs were pretreated with a miR-92a inhibitor for $48 \mathrm{~h}$. The results show that miR-92a expression was significantly increased compared with the negative control (NC), but decreased significantly after treatment with 

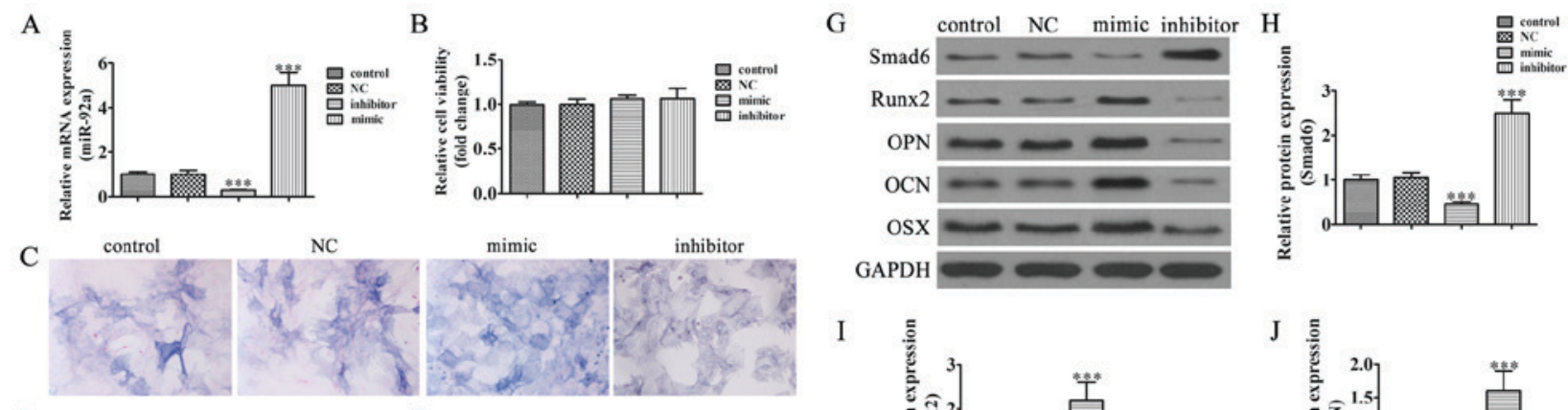
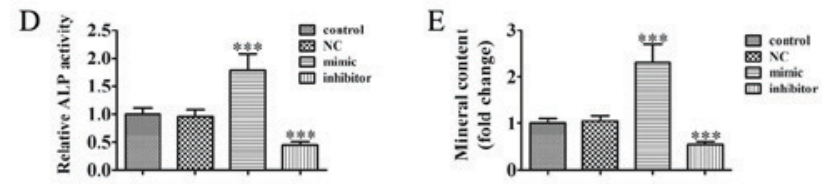

I

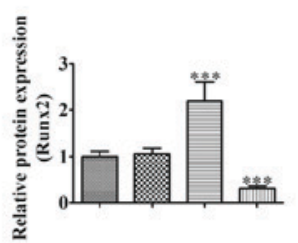

K
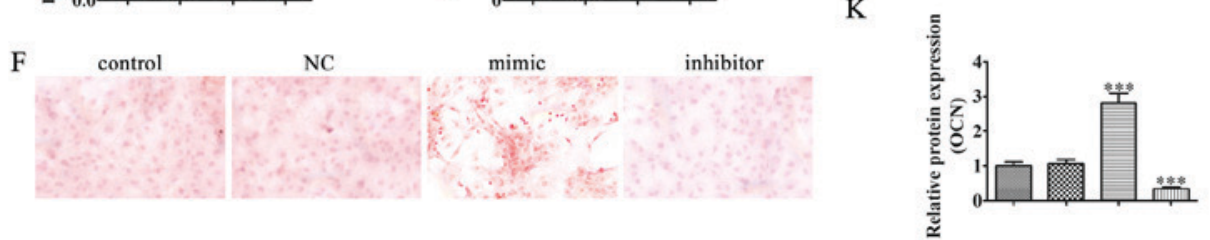

$\mathrm{J}$

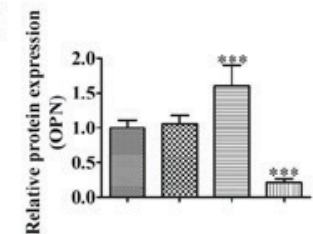

$\mathrm{L}$

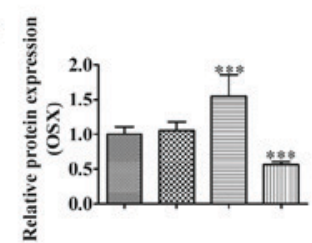

Figure 2. Expression of miR-92a promotes the osteogenesis of bone mesenchymal stem cells (BMSCs). (A) RT-qPCR shows miR-92a expression after transfection with miR-92a mimics or treatment with miR-92a inhibitor for $48 \mathrm{~h}$. (B) Cell viability was determined with an MTT assay. Data are presented as the mean $\pm \mathrm{SD},(\mathrm{n}=5)$. (C and D) Alkaline phosphatase (ALP) histochemical staining assay showing the effect of miR-92a on the osteogenic ability of BMSCs after being induced in osteogenic differentiation media for 21 days. ALP activities were evaluated by spectrophotometry. Data are presented as the mean \pm SD ${ }^{* * *} \mathrm{P}<0.001$ vs. the untreated control $(\mathrm{n}=5$ ). Magnification, $\mathrm{x} 400$. ( $\mathrm{E}$ and $\mathrm{F}$ ) Mineralized bone matrix formation was evaluated by Alizarin Red staining after BMSCs were induced in osteogenic differentiation media for 21 days. Mineralized nodule formation was assessed by Alizarin red staining. The stains were eluted and measured at $590 \mathrm{~nm}$. Data are presented as the mean $\pm \mathrm{SD} .{ }^{* * *} \mathrm{P}<0.001 \mathrm{vs}$. the untreated control $(\mathrm{n}=5)$. Magnification, $\mathrm{x} 200$. (G) Western blot assessment of Smad6, Runx2, OPN, OCN, and OSX expression. (H-L) Quantification of relative protein expression. Data are presented as the mean \pm SD. ${ }^{* * *} \mathrm{P}<0.001$ vs. the untreated control $(\mathrm{n}=3)$. $\mathrm{NC}$, negative control; Runx2, runt-related transcription factor 2; OPN, osteopontin; OCN, osteocalcin; OSX, osterix.

a miR-92a inhibitor (Fig. 2A). The MTT assay showed that both miR-92a overexpression and downregulation have not significantly affected cell activity (Fig. 2B).

To identify if miR-92a expression can promote the osteogenic differentiation, BMSCs transfected with miR-92a mimic or miR-92a-NC, or treated with a miR-92a inhibitor were induced in osteogenic differentiation media for 21 days. Then ALP activity (Fig. 2C and D) and Alizarin Red S staining (Fig. 2E and F) were used to detect osteogenic differentiation. The results show that miR-92a overexpression significantly increased ALP activity and calcium deposition compared with the control group. However, the ALP activity and calcium deposition were decreased after treatment with a miR-92a inhibitor.

Western blot detection showed that miR-92a expression suppressed Smad6 expression, which increased Runx 2 expression indirectly. Previous studies have found that Runx2 is upstream of OSX in the osteogenic differentiation signaling pathway $(15,16)$. Our study also verified that miR-92a overexpression promotes OCN, OPN, and OSX expression. However, miR-92a-specific inhibitors suppressed Runx 2 expression by promoting Smad6, which then inhibited the relative protein expression of osteogenic differentiation (Fig. 2G-L).

Smad6 overexpression reversed miR-92a induced osteogenic differentiation of BMSCs. To identify if the promotion effect of miR-92a on the osteogenic differentiation of BMSCs is by downregulating Smad6, a Smad6 overexpression vector was constructed and transfected into BMSCs. BMSCs were cultured for $48 \mathrm{~h}$ and then analyzed with western blot (Fig. 3A) and RT-qPCR (Fig. 3B). The results show that the expression of Smad6 was significantly increased at both the protein and mRNA level when Smad6 was overexpressed. After osteogenic differentiation was induced in media for 21 days, ALP activity (Fig. 3C and D) and Alizarin Red S staining (Fig. 3E and F) were used to detect osteogenic differentiation in BMSCs. The results show that Smad6 overexpression reversed miR-92a induced ALP activity and calcium deposition increased compared with the control group. Western blot detection showed that the expression of Smad6 decreased Runx2 expression. Our study also verified that Smad6 overexpression decreased miR-92a induced OCN, OPN, and OSX expression (Fig. 3G-L).

Smad6 is the direct target of miR-92a. To determine the possible interaction between miR-92a and Smad6, we first performed a bioinformatics screen for its possible target genes using an online 3'-UTR binding site prediction database (http://www.targetscan.org/). Overlap analyses showed that miR-92a had a broadly conserved binding site. A mutated version of the Smad6 3'-UTR was constructed in which six complementary nucleotides in the binding site were altered (Fig. 4A). This mutated construct was fused to the luciferase coding region (PYr-Smad6 3'-UTR) and co-transfected into HEK293T cells along with miR-92a mimics. The relative luciferase activity showed that when the wild-type Smad6 3'-UTR was co-transfected with miR-92a mimics, Smad6 expression was significantly decreased compared with co-transfection with the control miRNA (Fig. 4B). However, this effect was not observed after mutant 3'-UTR of Smad6, indicating that miR-92a can specifically target and suppress the 3'-UTR of Smad6. Western blot analyses further confirmed that miR-92a 
A

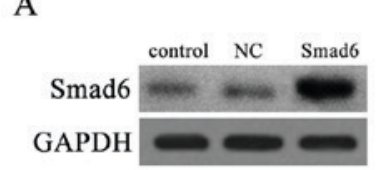

B

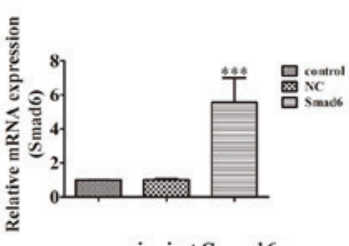

C

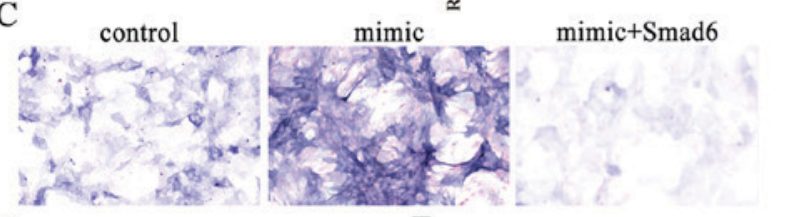

D

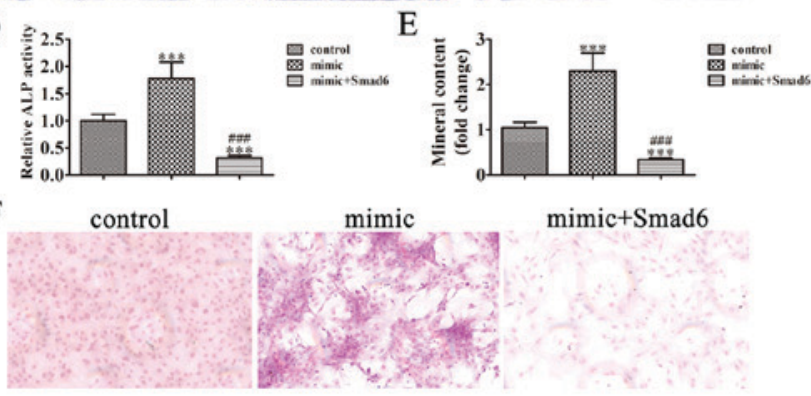

G

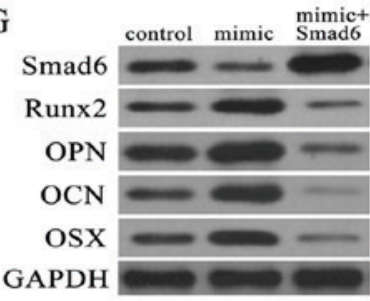

I

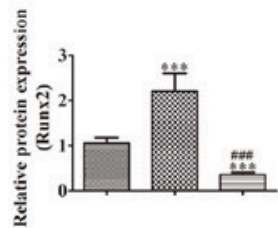

$\mathrm{K}$

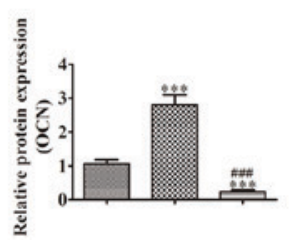

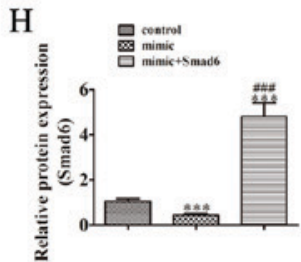

$\mathbf{J}$

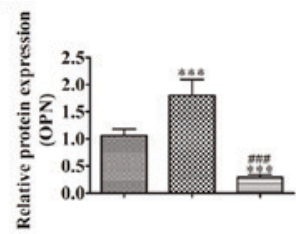

L

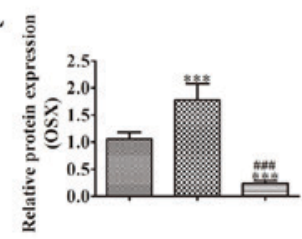

Figure 3. Smad6 overexpression reversed miR-92a induced osteogenesis of bone mesenchymal stem cells (BMSCs). (A) Western blotting and (B) RT-qPCR analysis show the expression of Smad6 in BMSCs cells after transfection with the Smad6 overexpression vector or the NC vector for $48 \mathrm{~h}$. Data are presented as the mean $\pm \mathrm{SD} .{ }^{* * *} \mathrm{P}<0.001$ vs. control $(\mathrm{n}=5)$. (C and $\left.\mathrm{D}\right)$ Alkaline phosphatase (ALP) histochemical staining assay showing the effect of miR-92a on the osteogenic ability of BMSCs. ALP activities were evaluated with spectrophotometry. Data are presented as the mean \pm SD. ${ }^{* * *} \mathrm{P}<0.001 \mathrm{vs}$. the untreated control.

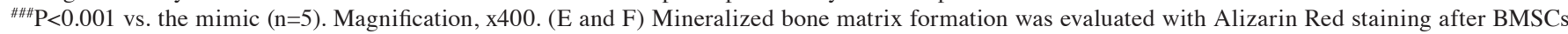
were induced in osteogenic differentiation media for 21 days. Mineralized nodule formation was assessed with Alizarin red staining. The stains were eluted and measured at $590 \mathrm{~nm}$. Data are presented as the mean $\pm \mathrm{SD}$. ${ }^{* * *} \mathrm{P}<0.001 \mathrm{vs}$. the untreated control. ${ }^{\# \# \#} \mathrm{P}<0.001$ vs. the mimic $(\mathrm{n}=5)$. Magnification, $\mathrm{x} 200$. (G) Western blot assessment of the expression of Smad6, Runx2, OPN, OCN, and OSX. (H-L) Quantification of relative protein expression. Data are presented as the mean $\pm \mathrm{SD} .{ }^{* * *} \mathrm{P}<0.001$ vs. the untreated control. ${ }^{\# \# "} \mathrm{P}<0.001$ vs. the mimic $(\mathrm{n}=3)$. NC, negative control; Runx2, runt-related transcription factor 2 ; OPN, osteopontin; OCN, osteocalcin; OSX, osterix.

A

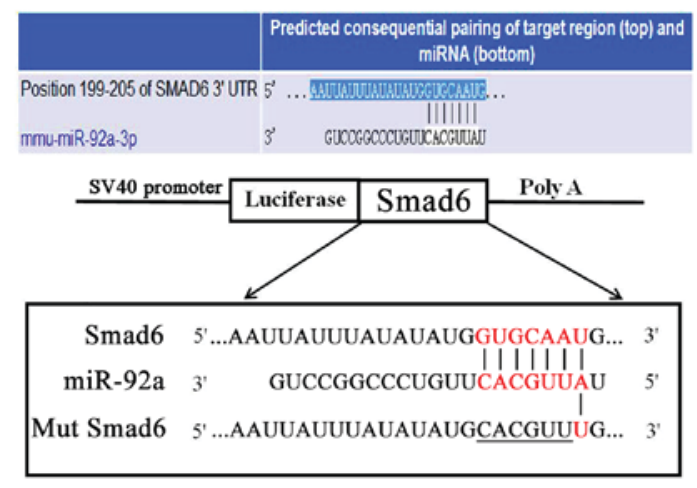

B

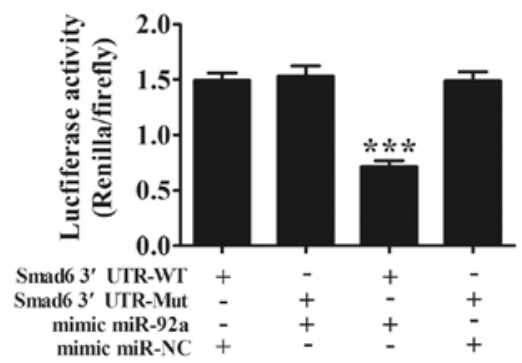

$\mathrm{C}$

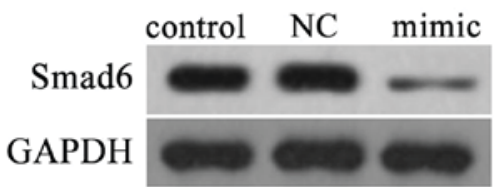

Figure 4. Smad6 is a potential target of miR-92a. (A) Complementary sequences between miR-92a and the 3'-UTR of Smad6 mRNA were obtained using publicly available algorithms. The mutated version of the Smad6 3'-UTR is also shown. (B) The 3'-UTR of Smad6 was fused to the luciferase coding region (PYr-Smad6 3'-UTR) and co-transfected into HEK293T cells with miR-92a mimics to confirm that RGS-17 is the target of miR-203. The PYr-Smad6 3'-UTR and miR-92a mimic constructs were co-transfected into HEK293T cells with a control vector and the relative luciferase activity was determined $48 \mathrm{~h}$ after transfection. The data are expressed as the mean $\pm \mathrm{SD}$. ${ }^{* * *} \mathrm{P}<0.001$ vs. the control. (C) Western blot analysis of Smad6 expression in BMSCs cells after transfection with miR-92a mimics $(\mathrm{n}=3)$. GAPDH expression levels were detected as an endogenous control.

expression significantly inhibited Smad6 expression in vitro (Fig. 4C).

\section{Discussion}

In this study, we investigated whether Smad6 could be used as a potential target to promote osteogenic differentiation of BMSCs. BMSCs were isolated from mice and a miR-92a overexpression vector was constructed. Our data have provided the evidence that overexpression of miR-92a promotes osteogenic differentiation of BMSCs by targeting Smad6. Shen et al have identified that Smad6 can interact with Runx2 and enhance Smurf1-induced Runx2 degradation in a ubiquitin-proteasome-dependent manner $(12,17)$. Runx 2 expression was also increased with miR-92a overexpression, which resulted in increased ALP activity and matrix mineralization capacity in BMSCs. 
Runx2 is an important osteoblast lineage-determining transcription factor involved in directing osteoblastic differentiation, and Runx 2 appears to be the master gene in osteogenesis as it induces the expression of OCN, OSX, and OPN, which are required in terminal osteoblast differentiation $(18,19)$. Our data strongly suggest that miR-92a plays a major role in osteogenic differentiation and functions in a Smad6-Runx2-dependent manner, adding a new layer of control to the regulation of the epigenetic program of osteogenesis. A previous report showed that miR-92a may have the potential to promote osteoblast proliferation and differentiation (20). However, our results show that Runx 2 expression was not affected by Smad6overexpression in a miR-92a mimic treatment group in BMSCs from mice, indicating that miR-92a can suppress Smad6 expression by targeting the 3'-UTR of Smad6 at the mRNA level. This result was confirmed with a bifluorescein reporting assay.

In conclusion, our in vitro study using transgenic BMSCs is the first demonstration that miR-92a can induce osteogenesis. Our findings are also consistent with the ability of miRNA-modified BMSCs to promote osteoblast differentiation by targeting Smad6. Although our results are very promising, further experimentation will be needed to investigate whether miRNA-modified BMSCs could also promote osteogenesis with miR-92a-modified BMSCs in vivo. Despite these limitations, our study supports the therapeutic promise of miR-92a in promoting osteogenic differentiation.

\section{Acknowledgements}

Not applicable.

\section{Funding}

Not applicable.

\section{Availability of data and materials}

The datasets used and/or analyzed during the current study are available from the corresponding author on reasonable request.

\section{Authors' contributions}

XY and HW designed the study and wrote the paper. YL and WH analyzed the data and wrote the paper. YJ and QS performed the experiments.

\section{Ethics approval and consent to participate}

All study protocols were approved by the Scientific Research Projects Approval Determination of the Ethics Committee of the 455th Hospital of PLA.

\section{Consent for publication}

Not applicable.

\section{Competing interests}

The authors declare that they have no competing interests.

\section{References}

1. Zheng YH, Xiong W, Su K, Kuang SJ and Zhang ZG: Multilineage differentiation of human bone marrow mesenchymal stem cells in vitro and in vivo. Exp Ther Med 5: 1576-1580, 2013.

2. Zhang L, Tang Y, Zhu X, Tu T, Sui L, Han Q, Yu L, Meng S, Zheng L, Valverde P, et al: Overexpression of MiR-335-5p promotes bone formation and regeneration in mice. J Bone Miner Res 32: 2466-2475, 2017.

3. Kim MJ, Park JS, Kim S, Moon SH, Yang HN, Park KH and Chung HM: Encapsulation of bone morphogenic protein-2 with Cbfa1-overexpressing osteogenic cells derived from human embryonic stem cells in hydrogel accelerates bone tissue regeneration. Stem Cells Dev 20: 1349-1358, 2011.

4. Kidwai F, Edwards J, Zou L and Kaufman DS: Fibrinogen induces RUNX2 activity and osteogenic development from human pluripotent stem cells. Stem Cells 34: 2079-2089, 2016.

5. Wang C, Liu D, Zhang C, Sun J, Feng W, Liang XJ, Wang S and Zhang J: Defect-related luminescent hydroxyapatite-enhanced osteogenic differentiation of bone mesenchymal stem cells via an ATP-induced cAMP/PKA pathway. ACS Appl Mater Interfaces 8: 11262-11271, 2016.

6. Inada M, Yasui T, Nomura S, Miyake S, Deguchi K, Himeno M, Sato M, Yamagiwa H, Kimura T, Yasui N, et al: Maturational disturbance of chondrocytes in Cbfa1-deficient mice. Dev Dyn 214: 279-290, 1999.

7. Kim IS, Otto F, Zabel B and Mundlos S: Regulation of chondrocyte differentiation by Cbfa1. Mech Dev 80: 159-170, 1999.

8. Enomoto H, Enomoto-Iwamoto M, Iwamoto M, Nomura S, Himeno M, Kitamura Y, Kishimoto T and Komori T: Cbfa1 is a positive regulatory factor in chondrocyte maturation. J Biol Chem 275: 8695-8702, 2000 .

9. Takeda S, Bonnamy JP, Owen MJ, Ducy P and Karsenty G: Continuous expression of Cbfal in nonhypertrophic chondrocytes uncovers its ability to induce hypertrophic chondrocyte differentiation and partially rescues Cbfa1-deficient mice. Genes Dev 15: 467-481, 2001.

10. Ueta C, Iwamoto M, Kanatani N, Yoshida C, Liu Y, Enomoto-Iwamoto M, Ohmori T, Enomoto H, Nakata K, Takada K, et al: Skeletal malformations caused by overexpression of Cbfal or its dominant negative form in chondrocytes. J Cell Biol 153: 87-100, 2001.

11. Stricker S, Fundele R, Vortkamp A and Mundlos S: Role of Runx genes in chondrocyte differentiation. Dev Biol 245: 95-108, 2002.

12. Shen R, Chen M, Wang YJ, Kaneki H, Xing L, O'keefe RJ and Chen D: Smad6 interacts with Runx2 and mediates Smad ubiquitin regulatory factor 1-induced Runx2 degradation. J Biol Chem 281: 3569-3576, 2006.

13. Fan QM, Yue B, Bian ZY, Xu WT, Tu B, Dai KR, Li G and Tang TT: The CREB-Smad6-Runx2 axis contributes to the impaired osteogenesis potential of bone marrow stromal cells in fibrous dysplasia of bone. J Pathol 228: 45-55, 2012.

14. Zhang L, Chen P, Chen L, Weng T, Zhang S, Zhou X, Zhang B and Liu L: Inhibited Wnt signaling causes age-dependent abnormalities in the bone matrix mineralization in the Apert syndrome FGFR2(S252W/+) mice. PLoS One 10: e112716, 2015.

15. Chen S, Gluhak-Heinrich J, Wang YH, Wu YM, Chuang HH, Chen L, Yuan GH, Dong J, Gay I and MacDougall M: Runx2, osx, and dspp in tooth development. J Dent Res 88: 904-909, 2009.

16. Du J, Wang Q, Yang P and Wang X: FHL2 mediates tooth development and human dental pulp cell differentiation into odontoblasts, partially by interacting with Runx2. J Mol Histol 47: 195-202, 2016.

17. Lee YS, Park JS, Kim JH, Jung SM, Lee JY, Kim SJ and Park SH: Smad6-specific recruitment of Smurf E3 ligases mediates TGF- $\beta 1$-induced degradation of MyD88 in TLR4 signalling. Nat Commun 2: 460, 2011.

18. Ducy P, Zhang R, Geoffroy V, Ridall AL and Karsenty G: Osf2/Cbfa1: A transcriptional activator of osteoblast differentiation. Cell 89: 747-754, 1997.

19. Komori T: Runx2, a multifunctional transcription factor in skeletal development. J Cell Biochem 87: 1-8, 2002.

20. Li Y, Kong D, Ahmad A, Bao B and Sarkar FH: Targeting bone remodeling by isoflavone and 3,3'-diindolylmethane in the context of prostate cancer bone metastasis. PLoS One 7: e33011, 2012 . 